\title{
Role of microRNAs in the process of metformin treating multiple diseases
}

\author{
Ningning $\mathrm{Ma}^{1,2,3}$, Jing Chen ${ }^{1,3^{*}}$, Jin $\operatorname{Ren}^{1,2,3^{*}}$
}

\begin{abstract}
Metformin as the first-line treatment for type 2 diabetes mellitus has been discovered to exert beneficial effects on many diseases for nearly ten years, but its specific mechanism is still unclear. As a new class of gene expression regulators with pleiotropic properties, microRNAs (miRNAs) participate in multiple physiological processes such as cell differentiation, proliferation, survival, and metabolism, which drive them to play a regulatory role in the occurrence, development and even treatment of various diseases. A substantial body of research has found the relationship between metformin and miRNAs, in which metformin can alter the expression profiles of miRNAs in multiple disease states and on the other hand the signal pathways involving miRNAs may contribute to the pharmacological actions of metformin. This review summarizes the effects of metformin on miRNAs and their relationship in different diseases (like tumor, metabolic diseases, etc.), which should be of a great help for our better understanding of the mechanism of metformin for treating multiple diseases.
\end{abstract}

\section{Keywords}

metformin; microRNA; tumor; metabolic diseases

Received 28 January 2021 accepted 24 April 2021

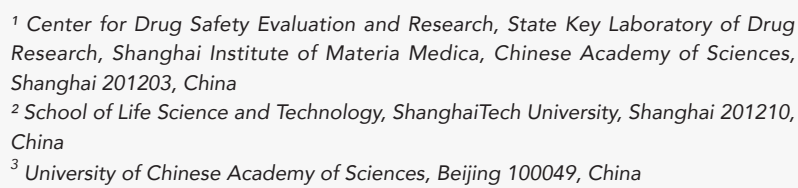

1 Center for Drug Safety Evaluation and Research, State Key Laboratory of Drug Research, Shanghai Institute of Materia Medica, Chinese Academy of Sciences, Shanghai 201203, China

${ }^{2}$ School of Life Science and Technology, ShanghaiTech University, Shanghai 201210 China

${ }^{3}$ University of Chinese Academy of Sciences, Beijing 100049, China

*Corresponding author Jing Chen, E-mail: jingchen@simm.ac.cn; Jin Ren, E-mail: jren@cdser.simm.ac.cn

\section{Introduction}

Metformin is the first-line drug recommended by the 2005 IDF Global Diabetes Treatment Guidelines for the treatment of type 2 diabetes mellitus due to its extensive effects on glucose metabolism including inhibiting gluconeogenesis and glycogen breakdown, reducing liver glucose output, increasing glucose transport in peripheral tissues and improving insulin sensitivity[1]. Since the approval of metformin sustained-release tablet (Gehuazhi) for marketing in the United States in 2000, its indications have been gradually extended with continuous indepth research with discovery of many new pharmacological actions of metformin such as cancer suppressing, aging delaying, and obesity relieving effects, as well as intestinal flora regulating function[2]. Although the exact mechanisms of these actions are not yet fully understood, the ability of metformin to promote phosphorylation or activation of AMPactivated protein kinase (AMPK) is considered to be the core of its molecular mechanism[3]. AMPK acts as a major regulator of energy metabolism by affecting many signaling pathways, including glucose and lipid metabolism, mitochondrial biogenesis, autophagy, cell growth, and circadian rhythm[4], which might explain in part the pharmacological properties of metformin.
MicroRNAs (miRNAs) are a class of endogenous non-coding RNAs of 18-25 nucleotides in length existing in eukaryotes. Primary and precursor miRNA molecules undergo nuclear and cytoplasmic processing by endonucleases, Drosha and Dicer 1 , respectively, to produce mature miRNAs that execute their cellular function[5]. MiRNAs extensively and finely regulate gene expression by multiple modes, including post-transcriptional repression or activation via pairing their seed, non-seed or full sequences with the 3'-UTR or 5'-UTR of target gene mRNA and transcriptional repression or activation via interacting with the transcriptional regulatory elements including promoter region, long non-coding RNA (IncRNA) or promoter associated RNAs (pRNAs), etc[6-7]. The multiplicity of miRNAs actions determines the diversity and complex network of their target genes, which confers their important roles in controlling cell growth, development, differentiation, death, metabolism, and even the occurrence and development of the diseases[8].

Considering the role of metformin in regulating multiple pathways and the characteristics of network regulation of miRNAs, researchers are curious about the relationship between miRNAs and metformin. Metformin has been documented to change miRNA expression profiles via enhancing the expression of Dicer1 in 
patients, mouse model and cell lines[9]. Besides upregulating Dicer1 expression[10], metformin can also disrupt the interaction between AU-binding factor 1 (AUF1) and Dicer1 mRNA and stabilize Dicer1 mRNA[10], leading to accumulation of Dicer1 thereby increasing the overall level of miRNAs. Moreover, metformin can also upregulate the expression of miRNAs through activating AMPK mimicking the action of AICAR, an AMPK activator[11-12]. Furthermore, metformin can upregulate the expression of certain protein-coding genes and non-coding RNAs by epigenetic mechanisms, e.g. by inducing genome-wide DNA demethylation[13-14].

In the current review, we provide a comprehensive summary with the most updated information on the role of miRNAs in mediating the pharmacological effects of metformin against multiple diseases.

\section{2 miRNAs contributes to the antitumor property of metformin}

Tumor refers to a new organism formed by the proliferation of local tissue cells stimulated by various tumorigenic factors. Epithelialmesenchymal transition (EMT) is the process in which epithelial cells acquire a mesenchymal phenotype with enhanced ability to migrate and invade, which is accompanied by the loss of cell polarity and intercellular adhesion. EMT is a key step in a series of events leading to cancer metastasis and most cancer-related deaths, thereby having become a hot topic in research. Metastasis is a word used to describe the spread of cancer cells from the primary neoplasm to distant sites, and the deregulated growth of malignant cell is the main reason for the failure of tumor treatment because of the poor prognosis, treatment difficulties and frequent relapses[15]. Many transcriptional factors including Snail family transcriptional repressors 1 (Snai1) and 2 (Snai2 or Slug), zinc finger E-box binding homeobox 1 (Zeb1), and the twist family bHLH transcription factor 1 (Twist1)[16] participate in the regulation of EMT. Besides transcriptional factor, metabolic shifts in tumor cells including altered glucose, lipid, and amino acid metabolism are also involved in EMT progression[17]. A number of epidemiological studies[18] reported that metformin affords appreciable anti-cancer efficacy by reducing cancer incidence and mortality in diabetic patients, though the underlying molecular mechanisms remain yet to be elucidated. Several mechanisms have been proposed to explain the antitumor activity of metformin, such as activation of the LKB1/AMPK pathway, induction of cell cycle arrest and/or apoptosis, inhibition of protein synthesis, repression of unfolded protein responses, activation of the immune system, and possible eradication of cancer stem cells[19]. Additionally, metformin was demonstrated to change the expression profiles of miRNAs in a variety of cancer tissues and cells, suggesting that miRNAs may be involved in the antitumor effects of metformin. A number of miRNAs have been linked to the antitumor action of metformin, such as let7, miRNA-21, miRNA-222, miRNA-26a, miRNA-146a, miRNA-200, etc[20]. Of note, it appears that metformin alters the expression of different miRNAs in different types of cancers, which might account for the antitumor activity of the drug (Table 1 ).

Table 1 List of miRNAs regulated by metformin with various cancers

\begin{tabular}{|c|c|c|c|c|}
\hline Cancer & Related miRNAs & Metformin effects & Target genes & Cell line \\
\hline \multirow[t]{5}{*}{ Lung cancer } & miR-381 & Upregulate & YAP & A549, H1299, Calu6, H520 \\
\hline & miR-7 & Upregulate & AKT/mTOR & A549 \\
\hline & & & Erk, NF-kB & \\
\hline & miR-222 & Downregulate & p27,p57 & A549 \\
\hline & & & PTEN & $\mathrm{NCl}-\mathrm{H} 358$ \\
\hline \multirow[t]{7}{*}{ Pancreatic cancer } & miR-26a & Upregulate & HMGA1 & Sw1990 \\
\hline & & & & Panc1 \\
\hline & $\mathrm{miR}-200 \mathrm{~b}$ & Upregulate & & AsPC-1 \\
\hline & & & & AsPC-1-GTR \\
\hline & & & & MiaPaCa-2 \\
\hline & miR-34a & Upregulate & Notch, Slug & Panc02 \\
\hline & miR-150 & Upregulate & & \\
\hline \multirow[t]{5}{*}{ Breast cancer } & miR-21-5p & Downregulate & SESN1 & SUM159PT \\
\hline & & & CAB39L & MCF-7, BT-474 \\
\hline & & & & BT-549 \\
\hline & miR-27a & Downregulate & AMPKa2 & MCF-7 \\
\hline & miR-26a & Upregulate & PTEN & MDA-MB-231 \\
\hline
\end{tabular}




\begin{tabular}{|c|c|c|c|c|}
\hline & & & MCL-1 & MDA-MB-468 \\
\hline & & & MTDH & MCF-7 \\
\hline & & & $\mathrm{EZH} 2$ & \\
\hline & miR-155 & Downregulate & & \\
\hline & miR-200c & Upregulate & C-Myc & MDA-MB-231 \\
\hline & & & AKT/Bcl-2 & MCF-7 \\
\hline & Let-7a & Upregulate & & \\
\hline & miR-96 & & & \\
\hline & miR-181a & Downregulate & & \\
\hline & miR-193b & Upregulate & FASN & MDA-MB-231 \\
\hline & & & & MDA-MB-468 \\
\hline & & & & BT-549 \\
\hline Renal cell & miR-26a & Upregulate & $\mathrm{Bcl}-2$ & $786-0$ \\
\hline carcinoma & & & Cyclin D1 & \\
\hline & miR-21 & Downregulate & PTEN & CAKI-1 \\
\hline & & & & CAKI-2 \\
\hline Oral Cancer & miR-26a & Upregulate & Mcl-1 & KB cell \\
\hline Skin cancer & miR-21 & Downregulate & PTEN/AKT & HaCaT \\
\hline Colon cancer & miR-21 & Downregulate & PTEN/AKT & HuTu80 \\
\hline & miR-1915 & Upregulate & Bcl-2 & HuTu80 \\
\hline Cervical cancer & miR-142-3p & Upregulate & HMGA2 & $\mathrm{SiHa}$ \\
\hline & & & & HeLa \\
\hline Esophageal & miR-497 & Upregulate & PELP1 & ESCC \\
\hline squamous cell & & & & \\
\hline carcinoma & & & & \\
\hline Osteosarcom a & miR-570-3p & Upregulate & LCMR1 & 143B \\
\hline & & & ATG12 & U2OS \\
\hline
\end{tabular}

\subsection{Lung cancer}

Lung cancer can be divided into two categories: small cell lung cancer accounting for $15-20 \%$ of cases and non-small cell lung cancer (NSCLC) accounting for $80-85 \%$ of cases according to the pathological characteristics and clinical manifestations. Metformin has been reported to control the invasiveness of NSCLC by upregulating miR-381 which also has tumor suppressive effects in other cancer types, such as breast cancer[21], pancreatic cancer[22], cervical cancer[23], and gastric cancer[24]. Moreover, the miR-381/YAP/Snail signal axis has been revealed as a contributor to the regulation of EMT by metformin[25]. MiR-7, another miRNA upregulated by metformin, has a suppressive effect on EGFR-mediated incidence and development of lung cancer by targeting PI3KR3/AKT, Bcl-2,
IGF-1R, and other signaling pathways. Thus, it is considered a new prognostic biomarker and therapeutic target in a variety of human cancer cells[26]. An increase of miR-7 induced by AMPK in metformin-treated A549 cells inhibited the AKT/mTOR, Erk, and NF-KB signaling pathways[27]. These findings suggest that the inhibitory effects of metformin on the growth, migration, and invasion of A549 cells are likely associated with the upregulation of miR-7. miR-222 has been shown to promote cell proliferation in a variety of tumors, including breast cancer[28], ovarian carcinoma[29], and hepatocellular carcinoma[30]. Metformin was found to downregulate miR-222 level by acting on AMPK signaling, which might explain the suppressive effect of metformin on the proliferation of lung cancer cells[31]. 


\subsection{Pancreatic cancer}

It was reported that metformin increases the expression of miR26a, miR-192, and let-7c expression in pancreatic cancer (PC) cell lines Sw1990 and Panc1 cells. MiR-26a is a tumor suppressor, whose down-regulation has been observed in many cancers including hepatocellular carcinoma, lung cancer, breast cancer and pancreatic duct adenocarcinoma (main tumor type of pancreatic cancer)[32-37]. Metformin inhibits the metastasis of pancreatic cancer cells by upregulating miR-26a which in turn represses the expression of its target gene HMGA1[38]. MiR-200b is another miRNA with elevated levels in metformintreated pancreatic spheres formed by AsPC-1, AsPC-1-GTR, or MiaPaCa-2 cells[39]. MiR-200b belongs to the miR-200 family which is believed to be a tumor suppressor because of their inhibitory effect on EMT of various cancer types[40], and its upregulation might mediate the antitumor activity of metformin. $\mathrm{Ji}$ et al[41] reported that miR-34a was downregulated in PC and contributed to increased mortality of patients diagnosed with PC. The inhibitory effect of miR-34a on EMT was ascribed to the suppression of transforming growth factor- $\beta$ (TGF- $\beta$ ), a major regulator of EMT. Restoration of miR-34a reduces the expression of Snail and other EMT regulators such as Notch and Slug[42]. Moreover, miR-34a mediates the suppressive effects of metformin on EMT-related targets. In addition to miR-34, miR-150 is also upregulated by metformin which contributes to the inhibitory effect of metformin on the proliferation of human pancreatic cancer cells, as a tumor suppressor miRNA[43].

\subsection{Breast cancer}

Like in other cancers, metformin also alter the expression profile of miRNAs in breast cancer (BC). MiR-21-5p, a highly expressed miRNA in a variety of tumors[44-47], has been reported to be reduced by metformin treatment in cultured human breast cancer cells, mouse xenografts, and serum from breast cancer patients[48]. Metformin-induced ablation of miR-21-5p increases the expression of SESN1 and CAB39L, leading to the activation of AMPK and inhibition of mTOR[48]. Moreover, knockdown of miR-21-5p by a sequence-specific antagomir mimics the effects of metformin on clonogenicity, migration, and invasiveness of multiple breast cancer cell lines[48]. MiR-27a is located on chromosome 19 and highly expressed as a carcinogenic miRNA in breast, gastric, pancreatic, and colon cancer[49]. Metformin treatment reduces the expression of miR-27a and upregulates the expression of AMPKa2 to elicit an anti-proliferative effect in MCF-7 cells[50]. In addition, metformin also upregulates miR-26a to repress its direct target genes BCL2, EZH2, and PTEN in breast cancer cells[51]. However, only PTEN and EZH2 expression reduction can be restored by miR$26 \mathrm{a}$ inhibition, suggesting the contribution of the metformin/miR26a/PTEN and EZH2 axis to the antitumor activity of metformin[51].
Similarly, metformin targeting the c-Myc/miR-200c/AKT2/Bcl-2 axis has also been identified as part of the mechanisms for its antitumor effect on breast cancer[52]. Apart from these, downregulation of carcinogenic miRNAs miR-155 and miR-181a and upregulation of tumor suppressor miRNAs let-7a and miR-96 by metformin may also be related to the inhibitory effect of metformin on breast cancer cell proliferation and/or migration[53-54].

Triple-negative breast cancer (TNBC) accounts for $10.0 \%$ to $20.8 \%$ of all breast cancers, with unique biological behavior and pathological characteristics rendering their prognosis worse than other types. Metformin upregulates miR-193a-3p and miR-193b in TNBC cells, of which, miR-193b can cause cell death and reduce mammosphere formation by TNBC cell lines via targeting FASN, one of the key regulators for the survival of TNBC[55]. Moreover, antagonizing miR-193 abrogates the decreasing action of metformin on FASN to cause cell death.

\subsection{Other cancers}

The modulation of miRNAs by metformin in cancers has been well recognized and the interactions between metformin and miRNAs are not limited to lung cancer, pancreatic cancer, and breast cancer. In 2014, Yang et al. demonstrated that metformin upregulated miR-26a, which subsequently repressed the expression of its target genes such as Bcl-2 and Cyclin D1 and inhibited the growth of human renal clear cell cancer cells (786-O)[56]. In addition, metformin can also induce apoptosis of human oral cancer cells by repressing the expression of $\mathrm{Mcl}-1$ via increasing miR26a[57]. Intriguingly, miR-21 seems to determine the differential sensitivities between human renal clear cell carcinoma cell lines CAKI-1 and CAKI-2 to metformin treatment: miR-21 enriched CAKI1 cells are more sensitive to metformin, whereas CAKI- 2 cells with lower abundance of miR-21 are insensitive to metformin[58]. Other studies demonstrated that the miR-21/PTEN/AKT signaling pathway contributes to the inhibitory effects of metformin on the viability of $\mathrm{HaCaT}$ cells in skin cancer[59] and HuTu80 cells in colon cancer[60]. In addition, metformin upregulates miR-1246 in gastric cancer, esophageal squamous cell carcinoma, and hepatocellular carcinoma cell lines, suggesting that miR-1246 may be related to the antitumor effects of metformin in various cancers[61].

Besides, some miRNAs are modulated by metformin only in a particular tumor based on the reported studies. For example, in colorectal cancer, miR-1915 is the most significantly up-regulated miRNA in HuTu80 cells treated with metformin (17 miRNAs are significantly up-regulated and 33 are down-regulated), which targets the anti-apoptotic protein $\mathrm{Bcl}-2$ and regulates multidrug resistance[62]. In cervical cancer, metformin inhibits the migration and invasion of cervical cancer cells by disrupting the MALAT1/ miR-142-3p sponge-like interaction to downregulate HMGA2 
expression[63]. In esophageal squamous cell carcinoma, metformin down-regulates PELP1 by up-regulating miR-497 leading to pyroptosis, which may be an alternative way to counter the apoptosis-resistant cancer[64]. In metastatic osteosarcoma, metformin increases miR-570-3p expression through DNA demethylation and inhibits translation of its target genes LCMR1 (a lung cancer metastasis-associated protein) and ATG12. Since high levels of autophagy is indicative of aggressiveness, low sensitivity to chemotherapy, and poor survival in some metastatic tumors, the effect of metformin on miR-570-3p/ATG12 is also associated with metformin-induced osteosarcoma cell metastasis and autophagy inhibition[65]. In hepatocellular carcinoma (HCC), 51 miRNAs were identified as differentially expressed miRNAs (33 upregulated and 18 downregulated) in HCC cell lines, in which the upregulated let-7 family may play a part in the suppression of HCC cell proliferation by metformin. Interestingly, the miRNAs upregulated by metformin include not only those that are overexpressed in HCC (such as miR-23a, miR-27a, miR$34 a$, miR-21, miR-24, and miR-324-5p), but also those that are reduced in HCC (such as miR-7a, miR-7b and miR-7e, miR-23b, miR-29a, miR-26a, miR-81a, miR-142-3p, and miR-378)[66].

In conclusion, it is generally accepted that metformin can change the expression profiles of miRNAs in various tumor tissues and cells. However, these changes are not consistent: some are common to many tumors, and others are specific to only one type of tumor. This may be related to the relative abundance of miRNA in different tumors. Additionally, the cellular functions of a given miRNA that is modulated in the same direction by metformin could be distinct in different tumors, which might be related to the relative abundance of the miRNA targets in different tumors.

\section{3 miRNAs are involved in the function of metformin against glycolipid metabolic disease}

Glucolipid metabolic disease (GLMD) refers to a complex of interrelated disorders in glucose and lipid metabolism, including obesity, type 2 diabetes mellitus (T2DM), hypertension, dyslipidemia, nonalcoholic fatty liver disease, and atherosclerosis, whose pathogenesis is extremely intricate[67]. As the major sources of energy and the key component of organism, the homeostasis of glucose, fatty acids and cholesterol are crucial to maintain the normal physiological function. Since glucose and lipid metabolism is intertwined, hyperglycemia, hyperlipidemia, hypertension and atherosclerosis are often concurrent in clinical[68]. Several studies have revealed that miRNAs play a pivotal role for controlling metabolic homoeostasis. MiR-122, the most abundant miRNA in the liver, has been shown to be involved in hepatic cholesterol and lipid metabolism[69]. As a firstline treatment for diabetes, metformin can regulate the body's metabolic level through a variety of mechanisms. The changes of miRNA expression in insulin-sensitive tissues of obese and/or T2DM patients have been discovered, suggesting that miRNAs may also play a role in mediating the effects of metformin on metabolic-related diseases[70] (Table 2).

Metformin was reported to alter the plasma concentrations of four circulating miRNAs, among which miR-140-5p and miR-222 are reduced and miR-142-3p and miR-192 are increased in T2DM[71]. Extracellular vehicles (EVs) are a heterogeneous group of cell-derived particles comprising exosomes and microvesicles that encapsulate miRNAs and proteins. Ghai et al. compared the miRNA profiles in whole plasma, EV, and EV-depleted plasma between T2DM patients receiving or not receiving metformin, and found that most of the affected miRNAs came from EV packages[72]. It has been reported that exosomal miR-222 secreted by the gonadal white adipose tissue (gWAT) promotes obesity-induced insulin resistance, which is the main feature of T2DM[73]. Prattichizzo proposed that miRNA-containing extracellular vesicles could be harnessed to restore a 'physiological' signature capable of preventing or delaying the harmful systemic effects of T2DM[74]. However, how metformin participates in the

Table 2 List of miRNAs regulated by metformin for treating glycolipid metabolic disease

\begin{tabular}{|c|c|c|c|c|}
\hline Metabolic Disease & Related miRNA & Metformin effects & Target genes & Cell line \\
\hline \multirow[t]{7}{*}{ T2DM } & $m i R-140-5 p$ & Downregulate & & \\
\hline & miR-222 & Downregulate & & \\
\hline & miR-142-3p & Upregulate & & \\
\hline & miR-192 & Upregulate & & \\
\hline & $\operatorname{miR}-33 b$ & Downregulate & СРT1 & HepG2 \\
\hline & & & CROT & \\
\hline & miR-291-3b & Downregulate & AMPKa1 & NCTC1469 \\
\hline
\end{tabular}


regulation of miRNAs levels in EV to treat T2DM and other diseases requires further research to elucidate.

Regulation of miRNAs by metformin has also been documented in vivo animal models of non-alcoholic fatty liver disease (NAFLD) and in vitro cell-based experiments[75]. In a study using methionine- and choline-deficient diet-induced non-alcoholic steatohepatitis model, metformin was found to downregulate miR-376a, miR-127, miR-34a, miR-300, and miR-342-3p and upregulate miR-122, miR-194, miR-101b, and miR-705[76]. In a high-fat diet-induced NAFLD model, two miRNAs are upregulated (miR-702-3p and miR-7085-3p), and two miRNAs are downregulated (miR-199b-5p and miR-451a) after metformin treatment[77]. Moreover, metformin reduces the expression of miR-33a and miR-33b by $70 \%$ in high glucose-treated HepG2 cells. And overexpression of miR-33b significantly abrogates the metformin-induced reduction of blood lipid content by about $50 \%$ and the intercellular and extracellular TG level by $50 \%$ and $20 \%$, respectively. These findings indicate that metformin can attenuate high glucose-induced lipid accumulation in HepG2 cells by downregulating miR-33b expression and subsequently increasing fatty acid $\beta$-oxidation[78]. In addition, compounds that regulate AMPK can affect the biosynthesis of liver miRNAs by altering Dicer1 expression. The expression of miR-30b and miR-30c in NAFLD is reduced, and palmitic acid (PA) and compound $C$ which simulate lipid disruption decrease the expression of miR-30b and miR-30c in liver cells, while metformin, as an AMPK agonist, upregulates miR-30b expression. This suggests that metformin can regulate fatty acid metabolism and might be used for the treatment of NAFLD by affecting miR-30 family[79]. Furthermore, miR-291b-3p which is reduced by metformin promotes hepatic lipogenesis by suppressing AMPKa1 expression and activity[80].

Visceral adipose tissue dysfunction contributes to the pathophysiology of multiple metabolic syndromes. Metformin has been reported to inhibit adipogenesis in murine preadipocyte cell lines and induce a significant time-dependent increase of miR1246 and miR-3687 and decrease of miR-148b-3p, miR-378a$3 p$, miR-378i, and miR-422a. Based on the functionalities of these miRNAs in preadipocyte, it is reasonable to speculate that they likely mediate the inhibitory effect of metformin on preadipocyte differentiation[81].

Collectively, accumulating evidence supports the role of metformin in regulating the expression of miRNAs and their downstream target genes in the setting of glycolipid metabolic disease. Since miRNAs are critical regulators of fatty acid and cholesterol metabolism, glucose homeostasis, insulin secretion and corresponding signaling[82-83], modulation of these miRNAs is likely a new mechanism by which metformin regulates glucose and lipid metabolism.

\section{4 miRNAs participate in other functions of metformin}

In addition to regulating tumorigenesis and glycolipid metabolism activity, metformin also has many other pharmacological effects. This notion is supported by numerous studies on metformin and miRNAs (Table 3).

Metformin improves angiogenesis by increasing the expression of vascular endothelial growth factor A (VEGFA) and reducing angiogenesis inhibitors in CD34+ cells[84]. In T1DM, the plasma levels of anti-angiogenic miRNAs increase, such as miR-222,

Table 3 Lists of miRNAs involved in metformin treating other diseases

\begin{tabular}{|c|c|c|c|c|}
\hline Disease & Related miRNA & Metformin effects & Target genes & Cell line \\
\hline \multirow[t]{4}{*}{ Anti-angioge nesis } & miR-21 & Downregulate & PTEN, & HUVEC \\
\hline & & & SMAD7 & \\
\hline & $\mathrm{miR}-221 / 222$ & Upregulate & p27Kip1 & \\
\hline & miR-34a & Downregulate & Sirtuin1 & MMEC \\
\hline Atheroscleros is & $\operatorname{miR}-124$ & Downregulate & $\mathrm{P} 4 \mathrm{Ha} 1$ & VSMC \\
\hline \multirow[t]{6}{*}{ Inflammation } & miR-34a-5p & Upregulate & & RAW264.7 \\
\hline & $m i R-125 b-5 p$ & & & \\
\hline & $\mathrm{miR}-146 a$ & Upregulate & & \\
\hline & miR-99b & Upregulate & AKT & \\
\hline & miR-155 & Downregulate & AKT & \\
\hline & miR-206 & Downregulate & HDAC4/ cyclin D1 & \\
\hline
\end{tabular}


miR-195 and miR-21a, while the level of angiogenic miR-126 decreases. The cardioprotective effect of metformin is achieved at least partly by acting on these angiogenesis-regulating miRNAs[85]. MiR-21 is known to regulate angiogenesis and Luo et al. discovered that metformin treatment downregulated the expression of miR-21 to derepress PTEN and SMAD7 expression and enhance the PI3K/AKT pathway[86]. In another study involving diabetic patients with cardiovascular disease, miR221/222 expression levels were significantly increased, which resulted in repression of cyclin-dependent kinase inhibitor p27Kip1 leading to thickening of intimal arteries after arterial injury[87]. The same study also unraveled that the alterations of miR-221/222 and p27Kip1 in diabetic subjects treated with metformin were similar to those in non-diabetic subjects[87]. Besides, miR-34a has an antiangiogenic effect in mouse microvascular endothelial cells (MMECs) by regulating the expression of Sirtuin1[88]. Treating MMEC cells with metformin inhibits the expression of miR-34a, which led to the recovery of angiogenic capacity in MMECs under high glucose conditions. Therefore, miR-34a represents both a target for metformin to mediate its vascular protective effect and a potential therapeutic target for the prevention/treatment of diabetic vascular disease[88]. Additionally, metformin activates AMPKa/ AP-2a/miR-124/P4Ha1 signaling in diabetic atherosclerosis, which plays a key role in the regulation of collagen synthesis in advanced atherosclerotic lesions[89]. This discovery provides an explanation for the observation that metformin increases the stability of atherosclerotic plaques in patients with diabetes[89].

To date, mounting evidence suggests that metformin has antiaging effects. Mice treated with metformin live longer and have a lower incidence of age-related chronic diseases. With aging, Dicer1 mRNA level decreases significantly, which indicates the relationship between miRNAs and aging. In mice treated with metformin, the expression of several miRNAs related to aging, including miR20a, miR-34a, miR-130a, miR-106b, miR-125 and let-7c are all increased[11]. Chronic and low-grade inflammation is one of the signs of insulin resistance caused by obesity, and metformin can produce anti-inflammatory effects in macrophages by inducing Dicer1 and upregulating miR-34a-5p and miR-125b-5p[90]. Hypertension damages many organs including blood vessels, heart, kidneys, brain, eyes, and leads to sexual dysfunction, bone loss, sleep apnea, etc. Metformin can improve cardiac fibrosis at the histopathological level by regulating AMPK, ameliorate cardiac hypertrophy at the molecular level, and reduce cardiovascular and renal insufficiency caused by hypertension[91]. Studies have shown that in an animal model of cardiac hypertrophy caused by deoxycorticosterone acetate-salt-induced hypertension, miR$146 a$, an anti-inflammatory miRNA, is significantly reduced, miR-155 that upregulates AKT is obviously increased, and miR99b that downregulates AKT is substantially decreased[92]. All these miRNAs are involved in regulating cardiac hypertrophy and their expression can be restored by metformin treatment, indicating that metformin plays a role in preventing myocardial hypertrophy by regulating these miRNAs[92]. Asthma is a chronic inflammatory airway disease characterized by overreaction and airway remodeling[93]. In cold areas, the body's immune function is declined, which can induce chronic bronchitis, asthma and other respiratory diseases[94]. Activation of AMPK regulates the miR-206/ HDAC4/cyclin D1 signaling pathway and has potential application in the prevention and treatment of asthma by reducing airway remodeling[95]. In addition, metformin also regulates miR-200 and miR-145 that directly target the major neural spine cell determinants Sox-1 and Sox-9[96].

With the discovery of the multiple therapeutic potentials of metformin and the complex characteristics of miRNA regulatory network, it is clear that metformin elicits multiple pharmacological actions by changing the expression of miRNAs, but the exact roles of the targeted miRNAs in mediating the therapeutic effects of metformin remain yet to be delineated.

\section{Conclusions and perspectives}

As the most widely used oral antidiabetic drug in the world, metformin not only lowers blood sugar, but also affords many other beneficial effects including antitumor and anti-aging activities. In view of the ability of metformin to modulate the expression of multiple miRNAs and the multiple functionalities of miRNAs, it is clear that miRNAs are one of the multiple mechanisms by which metformin produces its therapeutic efficacy against multiple human diseases. Here, we comprehensively summarize the progress in this area and discover that the change of miRNAs by metformin treatment is a shared mechanism for disease treatment. However, only a small number of miRNAs has been experimentally assessed and established for their mechanistic roles in conferring the therapeutic actions of metformin, and more experimental data are needed for consolidating the relationship between metformin and miRNAs.

It should be noted that the change of miRNAs is common and specific process in response to the treatment of metformin. Metformin can affect the expression of the same miRNAs such as miRNA-26a or miRNA-1246 in different diseases. In addition, due to the difference in the abundance of miRNAs in different tissues and cells, metformin can also change the expression of different miRNAs in the same disease. Recent studies have found that temperature also can affect miRNA profile. For example, altered ambient temperature can induce drastic but reversible changes in sequence length and abundance of both miRNA and piRNA populations in Drosophila[97]. High temperature stress has caused the fertility decline of many plants around the world, and one of causes is attributed to the change of miRNAs involved in almost all aspects of plant growth 
and development[98]. The future studies should be orientated to investigating the effects of metformin on various diseases under different temperature conditions in connection with the involvement of miRNAs.

Since the currently available research data were primarily obtained through in vitro cell and in vivo animal experiments, no relevant clinical studies on the subject have been reported. Therefore, well designed clinical trials for evaluating the relationships among metformin, diseases and miRNAs are encouraged. In addition, the miRNAs relevant to metformin could also be developed as small nucleic acid drugs for the treatment of related diseases, which could be another future research direction.

\section{Conflict of interests}

All authors declare no competing financial interest.

\section{Acknowledgments}

Supported by National Science \& Technology- ${ }^{* *}$ Major Project "Key New Drug Creation and Manufacturing Program", China (Grant No: 2018ZX09101001-003-007, 2018ZX09201017-004 and 2019ZX09732002-013)

\section{References}

[1] Sanchez-Rangel E, Inzucchi SE. Metformin: clinical use in type 2 diabetes. Diabetologia, 2017; 60(9): 1586-1593.

[2] Anisimov V N. Metformin for aging and cancer prevention. Aging, 2010; 2(11): 760-774.

[3] An H, He L. Current understanding of metformin effect on the control of hyperglycemia in diabetes. The Journal of Endocrinology, 2016; 228(3): R97-R106

[4] Hardie D G, Ross F A, Hawley S A. AMPK: a nutrient and energy sensor that maintains energy homeostasis. Nature Reviews. Molecular cell biology, 2012; 13(4): 251-262.

[5] Castellano L, Stebbing J. Deep sequencing of small RNAs identifies canonical and non-canonical miRNA and endogenous siRNAs in mammalian somatic tissues. Nucleic Acids Research, 2013; 41(5): 33393351.

[6] Pu M, Chen J, Tao Z, et al. Regulatory network of miRNA on its target: coordination between transcriptional and post-transcriptional regulation of gene expression. Cellular and Molecular Life Sciences: CMLS, 2019; 76(3): 441-451.

[7] Catalanotto C, Cogoni C, Zardo G. MicroRNA in Control of Gene Expression: An Overview of Nuclear Functions. International Journal of Molecular Sciences, 2016; 17(10): 1712.

[8] Kim Y K, Kim B, Kim V N. Re-evaluation of the roles of DROSHA, Export in 5, and DICER in microRNA biogenesis. Proceedings of the National Academy of Sciences of the United States of America, 2016; 113(13): e1881-e1889

[9] Blandino G, Valerio M, Cioce M, et al. Metformin elicits anticancer effects through the sequential modulation of DICER and c-MYC. Nature Communications, 2012; 3(1): 865.

[10] Abdelmohsen K, Tominaga-Yamanaka K, Srikantan S, et al. RNAbinding protein AUF1 represses Dicer expression. Nucleic Acids Research, 2012; 40(22): 11531-11544.

[11] Noren Hooten N, Martin-Montalvo A, Dluzen D F, et al. Metforminmediated increase in DICER1 regulates microRNA expression and cellular senescence. Aging Cell, 2016; 15: 572-581.

[12] Bridgeman S C, Ellison G C, Melton P E, et al. Epigenetic effects of metformin: From molecular mechanisms to clinical implications. Diabetes, Obesity \& Metabolism 2018; 20(7): 1553-1562.

[13] Zhong T, Men Y, Lu L, et al. Metformin alters DNA methylation genome-wide via the H19/SAHH axis. Oncogene, 2017; 36(17): 2345-
2354

[14] Yan L, Zhou J, Gao Y, et al. Regulation of tumor cell migration and invasion by the $\mathrm{H} 19 / \mathrm{let}-7$ axis is antagonized by metformin-induced DNA methylation. Oncogene, 2015; 34(23): 3076-3084

[15] Whyte F. Metastasis: the deadly part of cancer. British Journal of Nursing (Mark Allen Publishing), 1996; 5(9): 535-538.

[16] Park S M, Park S H, Ryu K J, et al. Downregulation of CHIP promotes ovarian cancer metastasis by inducing Snail-mediated epithelialmesenchymal transition. Molecular Oncology, 2019; 13(5): 1280-1295.

[17] Kang H, Kim H, Lee S, et al. Role of metabolic reprogramming in epithelial(-)mesenchymal transition (EMT). Int J Mol Sci, 2019; 20(8): 2042.

[18] Muti P, Berrino F, Krogh V, et al. Metformin, diet and breast cancer: an avenue for chemoprevention. Cell Cycle (Georgetown, Tex.), 2009; 8(16): 2661.

[19] Vona-Davis L, Rose D P. Type 2 diabetes and obesity metabolic interactions: common factors for breast cancer risk and novel approaches to prevention and therapy. Current Diabetes Reviews, 2012; 8(2): 116-130. [20] Zhou J Y, Xu B, Li L. A new role for an old drug: metformin targets MicroRNAs in treating diabetes and cancer. Drug Development Research, 2015; 76(6): 263-269.

[21] Xue Y, Xu W, Zhao W, et al. miR-381 inhibited breast cancer cells proliferation, epithelial-to-mesenchymal transition and metastasis by targeting CXCR4. Biomedicine \& Pharmacotherapy, 2017; 86: 426-433.

[22] Qiao G, Li J, Wang J, et al. miR-381 functions as a tumor suppressor by targeting ETS1 in pancreatic cancer. International Journal of Molecular medicine, 2019; 44(2): 593-607.

[23] Liu C, Tian X, Zhang J, et al. Long non-coding RNA DLEU1 promotes proliferation and invasion by interacting with miR-381 and enhancing HOXA13 expression in cervical cancer. Front Genet, 2018; 9: 629.

[24] Cao Q, Liu F, Ji K, et al. MicroRNA-381 inhibits the metastasis of gastric cancer by targeting TMEM16A expression. J Exp Clin Cancer Res, 2017; 36: 29.

[25] Jin D, Guo J, Wu Y, et al. Metformin-repressed miR-381-YAP-snail axis activity disrupts NSCLC growth and metastasis. J Exp Clin Cancer Res, 2020; 39(1): 6.

[26] Li M, Pan M, You C, et al. The therapeutic potential of miR-7 in cancers. Mini Reviews in Medicinal Chemistry, 2019; 19(20): 1707-1716.

[27] Dong J, Peng H, Yang X, et al. Metformin mediated microRNA-7 
upregulation inhibits growth, migration, and invasion of non-small cell lung cancer A549 cells. Anticancer Drugs, 2020; 31: 345-352.

[28] Chen W X, Hu Q, Qiu M T, et al. miR-221/222: promising biomarkers for breast cancer. Tumour Biology: The Journal of the International Society for Oncodevelopmental Biology and Medicine, 2013; 34(3): 1361-1370.

[29] Gong L, Zhang W, Yuan Y, et al. miR-222 promotes invasion and migration of ovarian carcinoma by targeting PTEN. Oncology Letters, 2018; 16(1): 984-990.

[30] Yang Y F, Wang F, Xiao J J, et al. MiR-222 overexpression promotes proliferation of human hepatocellular carcinoma HepG2 cells by downregulating p27. International Journal of Clinical and Experimental Medicine, 2014; 7(4): 893-902.

[31] Wang Y, Dai W, Chu X, et al. Metformin inhibits lung cancer cells proliferation through repressing microRNA-222. Biotechnol Lett, 2013; 35(12): 2013-2019.

[32] Deng J, He M, Chen L, et al. The loss of miR-26a-mediated posttranscriptional regulation of cyclin E2 in pancreatic cancer cell proliferation and decreased patient survival. PloS One, 2013; 8: e76450.

[33] Ren G, Li H, He X, et al. Downregulation of serum miR-26a predicts poor clinical outcome of papillary thyroid carcinoma. International Journal of Clinical And Experimental Pathology, 2017; 10(8): 9042-9047.

[34] Hwang J, Min B H, Jang J, et al. MicroRNA Expression Profiles in Gastric Carcinogenesis. Scientific Reports, 2018; 8(1): 14393.

[35] Taheriazam A, Bahador R, Karbasy S H, et al. Down-regulation of microRNA-26a and up-regulation of microRNA-27a contributes to aggressive progression of human osteosarcoma. Diagnostic Pathology, 2015; 10(1): 166

[36] Liang L, Zeng J H, Wang J Y, et al. Down-regulation of miR-26a-5p in hepatocellular carcinoma: a qRT-PCR and bioinformatics study. Pathology, Research and Practice, 2017; 213(12): 1494-1509.

[37] Yang Y, Zhang P, Zhao Y, et al. Decreased MicroRNA-26a expression causes cisplatin resistance in human non-small cell lung cancer. Cancer Biology \& Therapy, 2016; 17(5): 515-525.

[38] Wan D, Hua M, Liu T, et al. Evaluation and application of highway route scheme based on projection method. Journal of Chongqing Jiaotong University(Natural Science), 2012.

[39] Bao B, Wang Z, Ali S, et al. Metformin inhibits cell proliferation, migration and invasion by attenuating CSC function mediated by deregulating miRNAs in pancreatic cancer cells. Cancer Prev Res (Phila), 2012; 5(3): 355-364.

[40] Bracken C P, Khew-Goodall Y, Goodall G J. Network-based approaches to understand the roles of miR-200 and other microRNAs in cancer. Cancer Res, 2015; 75(13): 2594-2599.

[41] Ji Q, Hao X, Zhang M, et al. MicroRNA miR-34 inhibits human pancreatic cancer tumor-initiating cells. PLoS One, 2009; 4(8): e6816.

[42] Cifarelli V, Lashinger L M, Devlin K L, et al. Metformin and rapamycin reduce pancreatic cancer growth in obese prediabetic mice by distinct microrna-regulated mechanisms. Diabetes, 2015; 64(5): 1632-1642.

[43] Kato K, Iwama H, Yamashita T, et al. The anti-diabetic drug metformin inhibits pancreatic cancer cell proliferation in vitro and in vivo: Study of the microRNAs associated with the antitumor effect of metformin. Oncol Rep, 2016; 35(3): 1582-1592.

[44] Ganci F, Sacconi A, Bossel Ben-Moshe N, et al. Expression of TP53 mutation-associated microRNAs predicts clinical outcome in head and neck squamous cell carcinoma patients. Ann Oncol, 2013; 24: 3082-3088. [45] Sekar D, Krishnan R, Thirugnanasambantham K, et al. Significance of microRNA 21 in gastric cancer. Clinics and Research in Hepatology and Gastroenterology, 2016; 40(5): 538-545.
[46] Ghorbanmehr N, Gharbi S, Korsching E, et al. miR-21-5p, miR141-3p, and miR-205-5p levels in urine-promising biomarkers for the identification of prostate and bladder cancer. The Prostate, 2019; 79: 8895.

[47] Qu K, Zhang X, Lin T, et al. Circulating miRNA-21-5p as a diagnostic biomarker for pancreatic cancer: evidence from comprehensive miRNA expression profiling analysis and clinical validation. Scientific Reports, 2017; 7(1): 1692.

[48] Pulito C, Mori F, Sacconi A, et al. Metformin-induced ablation of microRNA 21-5p releases Sestrin-1 and CAB39L antitumoral activities. Cell Discov, 2017; 3: 17022

[49] Li X, Xu M, Ding L, et al. MiR-27a: a novel biomarker and potential therapeutic target in tumors. Journal of Cancer, 2019; 10: 2836-2848.

[50] Zhao W, Zhang X, Liu J, et al. miR-27a-mediated antiproliferative effects of metformin on the breast cancer cell line MCF-7. Oncol Rep, 2016; 36(6): 3691-3699.

[51] Cabello P, Pineda B, Tormo E, et al. The antitumor effect of metformin is mediated by mir-26a in breast cancer. International Journal of Molecular Sciences, 2016; 17: 1298.

[52] Zhang J, Li G, Chen Y, et al. Metformin Inhibits Tumorigenesis and Tumor Growth of Breast Cancer Cells by Upregulating miR-200c but Downregulating AKT2 Expression. Journal of Cancer, 2017; 8(10): 18491864

[53] Sharma P, Kumar S. Metformin inhibits human breast cancer cell growth by promoting apoptosis via a ROS-independent pathway involving mitochondrial dysfunction: pivotal role of superoxide dismutase (SOD). Cell Oncol (Dordr), 2018; 41(6): 637-650.

[54] Oliveras-Ferraros C, Cufi S, Vazquez-Martin A, et al. Micro(mi)RNA expression profile of breast cancer epithelial cells treated with the antidiabetic drug metformin: induction of the tumor suppressor miRNA let$7 a$ and suppression of the TGFbeta-induced oncomiR miRNA-181a. Cell Cycle (Georgetown, Tex.) 2011; 10: 1144-1151.

[55] Wahdan-Alaswad R S, Cochrane D R, Spoelstra N S, et al. Metformininduced killing of triple-negative breast cancer cells is mediated by reduction in fatty acid synthase via miRNA-193b. Hormones \& Cancer, 2014; 5(6): 374-389.

[56] Yang F Q, Wang J J, Yan J S, et al. Metformin inhibits cell growth by upregulating microRNA-26a in renal cancer cells. International Journal of Clinical and Experimental Medicine, 2014; 7(10): 3289-3296.

[57] Wang F, Xu J, Liu H, et al. Metformin induces apoptosis by microRNA26a-mediated downregulation of myeloid cell leukaemia-1 in human oral cancer cells. Mol Med Rep, 2016; 13(6): 4671-4676.

[58] Kalogirou C, Schafer D, Krebs M, et al. Metformin-Derived Growth Inhibition in Renal Cell Carcinoma Depends on miR-21-Mediated PTEN Expression. Urol Int, 2016; 96(1): 106-115.

[59] Deng Y, Ma W. Metformin inhibits HaCaT cell viability via the miR-21/ PTEN/Akt signaling pathway. Mol Med Rep, 2018; 17(3): 4062-4066. [60] Roy S, Yu Y, Padhye S B, et al. Difluorinated-curcumin (CDF) restores PTEN expression in colon cancer cells by down-regulating miR-21. PLoS One, 2013; 8(7): e68543.

[61] Fujihara S, Kato K, Morishita A, et al. Antidiabetic drug metformin inhibits esophageal adenocarcinoma cell proliferation in vitro and in vivo. Int J Oncol, 2015; 46(5): 2172-2180.

[62] Chiyo T, Kato K, Iwama H, et al. Therapeutic potential of the antidiabetic drug metformin in small bowel adenocarcinoma. Int J Oncol, 2017; 50(6): 2145-2153.

[63] Xia C, Liang S, He Z, et al. Metformin, a first-line drug for type 2 diabetes mellitus, disrupts the MALAT1/miR-142-3p sponge to decrease 
invasion and migration in cervical cancer cells. Eur J Pharmacol, 2018; 830: $59-67$.

[64] Wang L, Li K, Lin X, et al. Metformin induces human esophageal carcinoma cell pyroptosis by targeting the miR-497/PELP1 axis. Cancer Lett, 2019; 450: 22-31.

[65] Bao X, Zhao L, Guan H, et al. Inhibition of LCMR1 and ATG12 by demethylation-activated miR-570-3p is involved in the anti-metastasis effects of metformin on human osteosarcoma. Cell Death Dis, 2018; 9(6): 611.

[66] Miyoshi $\mathrm{H}$, Kato $\mathrm{K}$, Iwama $\mathrm{H}$, et al. Effect of the anti-diabetic drug metformin in hepatocellular carcinoma in vitro and in vivo. Int $\mathrm{J}$ Oncol, 2014; 45(1): 322-332.

[67] Ye D W, Rong X L, Xu A M, et al. Liver-adipose tissue crosstalk: a key player in the pathogenesis of glucolipid metabolic disease. Chinese Journal of Integrative Medicine, 2017; 23(6): 410-414.

[68] Guo J. Research progress on prevention and treatment of glucolipid metabolic disease with integrated traditional Chinese and Western medicine. Chinese Journal of Integrative Medicine, 2017; 23(6): 403-409.

[69] Vienberg S, Geiger J, Madsen S, et al. MicroRNAs in metabolism. Acta Physiologica (Oxford, England), 2017; 219: 346-361.

[70] Herrera B M, Lockstone H E, Taylor J M, et al. Global microRNA expression profiles in insulin target tissues in a spontaneous rat model of type 2 diabetes. Diabetologia, 2010; 53(6): 1099-1109.

[71] Ortega F J, Mercader J M, Moreno-Navarrete J M, et al. Profiling of circulating microRNAs reveals common microRNAs linked to type 2 diabetes that change with insulin sensitization. Diabetes Care, 2014; 37(5): 1375-1383.

[72] Ghai V, Kim T K, Etheridge A, et al. Extracellular vesicle encapsulated microRNAs in patients with type 2 diabetes are affected by metformin treatment. J Clin Med, 2019; 8(5):617.

[73] Li D, Song H, Shuo L, et al. Gonadal white adipose tissue-derived exosomal MiR-222 promotes obesity-associated insulin resistance. Aging, 2020; 12(22): 22719-22743.

[74] Prattichizzo F, Giuliani A, De Nigris V, et al. Extracellular microRNAs and endothelial hyperglycaemic memory: a therapeutic opportunity? Diabetes, Obesity \& Metabolism, 2016; 18(9): 855-867.

[75] Buzzetti E, Pinzani M, Tsochatzis E A. The multiple-hit pathogenesis of non-alcoholic fatty liver disease (NAFLD). Metabolism: Clinical and Experimental, 2016; 65(8): 1038-1048.

[76] Katsura A, Morishita A, Iwama $\mathrm{H}$, et al. MicroRNA profiles following metformin treatment in a mouse model of non-alcoholic steatohepatitis. International Journal of Molecular Medicine, 2015; 35(4): 877-884.

[77] Guo J, Zhou Y, Cheng Y, et al. Metformin-induced changes of the coding transcriptome and non-coding RNAs in the livers of non-alcoholic fatty liver disease mice. Cell Physiol Biochem, 2018; 45(4): 1487-1505.

[78] Zare M, Panahi G, Koushki M, et al. Metformin reduces lipid accumulation in HepG2 cells via downregulation of miR-33b. Arch Physiol Biochem, 2019; 5: 1-8.

[79] Latorre J, Ortega F J, Liñares-Pose L, et al. Compounds that modulate AMPK activity and hepatic steatosis impact the biosynthesis of microRNAs required to maintain lipid homeostasis in hepatocytes. EBioMedicine, 2020; 53: 102697.

[80] Meng X, Guo J, Fang W, et al. Liver MicroRNA-291b-3p promotes hepatic lipogenesis through negative regulation of adenosine 5'-monophosphate (AMP)-activated protein kinase alpha1. J Biol Chem, 2016; 291(20): 10625-10634

[81] Fujita K, Iwama H, Oura K, et al. Metformin-suppressed differentiation of human visceral preadipocytes: Involvement of microRNAs. International
Journal of Molecular Medicine, 2016; 38(4): 1135-1140.

[82] Flowers E, Froelicher E S, Aouizerat B E. MicroRNA regulation of lipid metabolism. Metabolism, 2013; 62(1): 12-20.

[83] Lynn F C. Meta-regulation: microRNA regulation of glucose and lipid metabolism. Trends Endocrinol Metab, 2009; 20(9): 452-459.

[84] Bakhashab S, Ahmed F W, Schulten H J, et al. Metformin improves the angiogenic potential of human $\mathrm{CD} 34(+)$ cells co-incident with downregulating CXCL10 and TIMP1 gene expression and increasing VEGFA under hyperglycemia and hypoxia within a therapeutic window for myocardial infarction. Cardiovasc Diabetol, 2016; 15(1): 27.

[85] Ahmed F W, Bakhashab S, Bastaman I T, et al. Anti-Angiogenic miR222, miR-195, and miR-21a Plasma Levels in T1DM are improved by metformin therapy, thus elucidating its cardioprotective effect: the MERIT study. Int J Mol Sci, 2018; 19.

[86] Luo M, Tan X, Mu L, et al. MiRNA-21 mediates the antiangiogenic activity of metformin through targeting PTEN and SMAD7 expression and PI3K/AKT pathway. Sci Rep, 2017; 7: 43427.

[87] Coleman C B, Lightell D J, Moss S C, et al. Elevation of miR-221 and -222 in the internal mammary arteries of diabetic subjects and normalization with metformin. Mol Cell Endocrinol, 2013; 374(1-2): 125129.

[88] Arunachalam G, Lakshmanan A P, Samuel S M, et al. Molecular interplay between microRNA-34a and sirtuin 1 in hyperglycemia-mediated impaired angiogenesis in endothelial cells: effects of metformin. J Pharmacol Exp Ther, 2016; 356(2): 314-323.

[89] Liang W J, Zhou S N, Shan M R, et al. AMPKalpha inactivation destabilizes atherosclerotic plaque in streptozotocin-induced diabetic mice through AP-2alpha/miRNA-124 axis. J Mol Med (Berl), 2018; 96(5): 403412.

[90] Luo X, Hu R, Zheng Y, et al. Metformin shows anti-inflammatory effects in murine macrophages through Dicer/microribonucleic acid-34a-5p and microribonucleic acid-125b-5p. J Diabetes Investig, 2020; 11(1): 101109.

[91] Rahman F, McEvoy J W. Dangers of overly aggressive blood pressure control. Curr Cardiol Rep, 2018; 20(11): 108.

[92] Amara V R, Surapaneni S K, Tikoo K. Metformin attenuates cardiovascular and renal injury in uninephrectomized rats on DOCAsalt: Involvement of AMPK and miRNAs in cardioprotection. Toxicol Appl Pharmacol, 2019; 362: 95-104.

[93] Manuyakorn W, Howarth P H, Holgate S T. Airway remodelling in asthma and novel therapy. Asian Pacific Journal of Allergy and Immunology, 2013; 31(1): 3-10.

[94] Cong X, Xu X, Zhang Y, et al. Temperature drop and the risk of asthma: a systematic review and meta-analysis. Environmental Science and Pollution Research International, 2017; 24(28): 22535-22546.

[95] Pan Y, Liu L, Li S, et al. Activation of AMPK inhibits TGF-beta1-induced airway smooth muscle cells proliferation and its potential mechanisms. Sci Rep, 2018; 8(1): 3624

[96] Banerjee P, Dutta S, Pal R. Dysregulation of wnt-signaling and a candidate set of miRNAs underlie the effect of metformin on neural crest cell development. Stem Cells, 2016; 34(2): 334-345.

[97] Fast I, Hewel C, Wester L, et al. Temperature-responsive miRNAs in Drosophila orchestrate adaptation to different ambient temperatures. RNA (New York, N.Y.), 2017; 23(9): 1352-1364.

[98] Lin $Y$, Zhang L, Zhao Y, et al. Comparative analysis and functional identification of temperature-sensitive miRNA in Arabidopsis anthers. Biochemical and Biophysical Research Communications, 2020; 532(1): 1-10. 